\title{
T GRAFTS WITH THE RIGHT INTERNAL THORACIC ARTERY TO LEFT INTERNAL THORACIC ARTERY VERSUS THE LEFT INTERNAL THORACIC ARTERY AND RADIAL ARTERY: FLOW DYNAMICS IN THE INTERNAL THORACIC ARTERY MAIN STEM
}

\author{
Olaf Wendler, MD ${ }^{\mathrm{a}}$ \\ Benno Hennen, $\mathrm{MD}^{\mathrm{b}}$ \\ Torsten Markwirth, $\mathrm{MD}^{\mathrm{b}}$ \\ Jochem König, MD \\ Dietmar Tscholl ${ }^{\mathrm{a}}$ \\ Qi Huang ${ }^{\text {a }}$ \\ Erfane Shahangi ${ }^{\mathrm{a}}$ \\ Hans-Joachim Schäfers, $\mathrm{MD}, \mathrm{PhD}^{\mathrm{a}}$ \\ Sponsor: Hans G. Borst, MD
}

Objective: Complete arterial coronary artery bypass grafting with 2 grafts can be achieved even in triple vessel disease by use of a $T$ configuration. There is still uncertainty whether the coronary flow reserve in the main stem of the left internal thoracic artery is sufficient to supply more than 1 anastomosed coronary vessel. Methods: Between March 1996 and February 1999, 251 patients with multivessel coronary artery disease underwent complete arterial revascularization with $\mathbf{T}$ grafts, using either the left internal thoracic artery with the free right internal thoracic artery graft $(n=73$, group I) or the left internal thoracic artery and radial artery $(n=178$, group II). A mean of 4.0 (group I) versus 4.3 (group II) coronary vessels were anastomosed per patient. One week (n $=92)$ and 6 months $(n=28)$ after the operation, flow was measured in the proximal left internal thoracic artery with a Doppler guide wire. Maximum flow was determined after injection of adenosine $(30 \mu \mathrm{g})$. Results: The in-hospital mortality was $2.7 \%$ (group I) versus $2.3 \%$ (group II). At angiography $(n=142,56.6 \%)$ the patency rate was $96.3 \%$ (group I) versus $98.2 \%$ (group II). There was no significant difference between baseline flow, maximum flow, and coronary flow reserve between the 2 groups. Coronary flow reserve increased in both groups within the first 6 postoperative months (group I, $1.85 \pm 0.31$ vs $2.77 \pm$ $0.77, P=.0002$; group II, $1.82 \pm 0.4$ vs $2.53 \pm 0.73, P=.009)$. Conclusion: Both variants of $\mathbf{T}$ grafts allow for complete arterial revascularization with good perioperative results. The flow reserve of the proximal internal thoracic artery is adequate for multiple coronary anastomoses irrespective of the choice of the second arterial graft. (J Thorac Cardiovasc Surg 1999;118:841-8)
T he internal thoracic artery (ITA) currently is the conduit of choice in coronary artery bypass grafting $(\mathrm{CABG})$ because of superior graft patency, reduced cardiac events, and enhanced short-term and long-term

From the Department of Thoracic and Cardiovascular Surgery, ${ }^{\mathrm{a}}$ Department of Cardiology, ${ }^{\mathrm{b}}$ Institution of Biometrics, ${ }^{\mathrm{c}}$ University Hospital Homburg/Saar, Germany.

Read at the Seventy-ninth Annual Meeting of The American Association for Thoracic Surgery, New Orleans, La, April 18-21, 1999.

Received for publication April 22, 1999; revisions requested June 15, 1999; revisions received July 8, 1999; accepted for publication Aug 3, 1999.

Address for reprints: Dr med Olaf Wendler, Abt. Thorax- und HerzGefäßchirurgie, Universitätskliniken des Saarlandes, Kirrberger Strasse 1, D-66421 Homburg/Saar, Germany (E-mail: chowen@med.rz.uni-sb.de).

Copyright (C) 1999 by Mosby, Inc.

$0022-5223 / 99 \$ 8.00+0 \quad \mathbf{1 2 / 6 / 1 0 1 9 1 8}$ survival. ${ }^{1-3}$ The use of both the left and free right ITA for revascularization of the anterior descending and circumflex coronary arteries has shown additional advantages over the use of only 1 ITA in combination with vein grafts with respect to long-term survival and quality of life, that is, freedom from angina and reintervention. ${ }^{2,4,5}$

Complete arterial revascularization appears to be the next logical step to optimize surgical therapy for coronary heart disease. In most of these operations, 3 or more arterial grafts are used for complete revascularization of triple vessel disease. The associated trauma and time required for this approach impede a more widespread use of complete arterial revascularization.

Tector and associates ${ }^{6}$ in 1994 presented the technique of $\mathrm{T}$ grafting for complete arterial revascularization with both ITAs. In this approach the left ITA is connected to the coronary branches of the anterior wall, 
Table I. Clinical characteristics of patients with $T$ grafts

\begin{tabular}{lllc}
\hline & $\begin{array}{c}\text { LITA-RITA } \\
(n=73)\end{array}$ & $\begin{array}{c}\text { LITA-RA } \\
(n=178)\end{array}$ & P value \\
\hline Mean age (y) & $61.4 \pm 10.5$ & $60.5 \pm 8.6$ & $>.2$ \\
Sex (M/F) & $65 / 8$ & $140 / 38$ & .02 \\
CAD & & & \\
$\quad$ One vessel (n) & 0 & $5(3 \%)$ & $>.2$ \\
$\quad$ Two vessels (n) & $3(4 \%)$ & $173(97 \%)$ & $>.2$ \\
$\quad$ Three vessels (n) & $70(96 \%)$ & $45(25 \%)$ & $>.2$ \\
Left main stenosis & $18(25 \%)$ & & \\
$\quad(\mathrm{n})$ & & & \\
Ejection fraction & $58.1 \pm 15.2(28-85)$ & $55.3 \pm 14.5(23-85)$ & $>.2$ \\
$\quad(\%)$ & & $2(1 \%)$ & $>.2$ \\
Preop IABP (n) & 0 & $14(8 \%)$ & $>.2$ \\
Redo cases (n) & $1(1 \%)$ & & \\
\hline
\end{tabular}

LITA, Left internal thoracic artery; RITA, right internal thoracic artery; $R A$, radial artery; $C A D$, coronary artery disease; $I A B P$, intra-aortic balloon pump.

whereas the second free ITA is anastomosed proximally to the left ITA and distally to the coronary arteries of the posterolateral and inferior wall in sequential fashion. T grafting with the left ITA and radial artery has also been reported..$^{7-9}$ With this approach, complete revascularization is possible with maximum graft economy (ie, 2 grafts) and reduction of operative trauma and time.

In 1996 we have adopted the approach of complete arterial revascularization with only 2 arterial grafts (either both ITAs or the left ITA and radial artery), commonly using the T-graft approach.

In both variants of this configuration, total coronary bypass flow is dependent on the flow of the left proximal ITA. This has led to concern whether flow reserve in the ITA main stem is sufficient, both in the perioperative and long-term phase.

We therefore studied blood flow in the ITA main stem of an arterial T-graft configuration using the left ITA graft and a free right ITA or left ITA and radial artery. The objective of these investigation was to compare baseline flow and maximum flow in the early postoperative period with the flow dynamics in a more long-term phase. The coronary flow reserve $(\mathrm{CFR}=$ ratio of maximum flow to baseline flow) was determined and compared 1 week and 6 months after the operation.

\section{Patients and methods}

Patients. During the period March 1996 to March 1999, 297 patients with multiple coronary artery disease underwent complete arterial revascularization in our institution. The decision for arterial revascularization was based on prognos-
Table II. Clinical characteristics and coronary risk factors of patients who underwent flow measurement studies 1 week and 6 months after the operation

\begin{tabular}{lccc}
\hline & $\begin{array}{c}\text { LITA-RITA } \\
(n=18)\end{array}$ & $\begin{array}{c}\text { LITA-RA } \\
(n=10)\end{array}$ & P value \\
\hline Mean age (y) & $58.4 \pm 13$ & $60.8 \pm 10.1$ & $>.2$ \\
Sex (M/F) & $16 / 2$ & $7 / 3$ & .2 \\
CAD & & & \\
$\quad 1$ vessel (n) & 0 & 0 & $>.2$ \\
2 vessels (n) & 0 & $1(10 \%)$ & $>.2$ \\
$\quad 3$ vessels (n) & $18(100 \%)$ & $9(90 \%)$ & $>.2$ \\
Ejection fraction (\%) & $61.5 \pm 10.9$ & $60.6 \pm 12.5$ & $>.2$ \\
Hypertension (n) & $8(44 \%)$ & $3(30 \%)$ & $>.2$ \\
Hyperlipidemia (n) & $11(61 \%)$ & $6(60 \%)$ & $>.2$ \\
Diabetes mellitus (n) & $4(22 \%)$ & $3(30 \%)$ & $>.2$ \\
Smoking history (n) & $7(39 \%)$ & $4(40 \%)$ & $>.2$ \\
\hline
\end{tabular}

LITA, Left internal thoracic artery; RITA, right internal thoracic artery; $R A$, radial artery; $C A D$, coronary artery disease.

tic reasons (age $\leq 70$ years) in $79 \%$ and lack of vein grafts in $21 \%$ of patients. In 251 (85\%) of these patients, revascularization was performed with a T-graft configuration. Clinical characteristics are shown in Table I.

Surgical technique. The radial artery and ITAs were prepared by means of the skeletonization technique. The radial artery was dissected at the nondominant arm by means of standard technique ${ }^{10}$ while the left ITA was harvested.

After preparation of both grafts (left ITA and free right ITA or left ITA and radial artery), diluted papaverine $(50 \mathrm{mg} / 20$ $\mathrm{mL}$ Ringer solution) was injected into the lumen of the arteries and the distal end of the grafts was closed with a Hemoclip device (Weck Closure Systems, Research Triangle Park, NC). ${ }^{11}$ The radial artery was additionally soaked in diluted diltiazem $(25 \mathrm{mg} / 20 \mathrm{~mL}$ Ringer solution). No routine systemic therapy with vasodilators or calcium channel blockers was given during the operation and the postoperative course.

All patients were operated on with extracorporeal circulation and mild systemic hypothermia $\left(32^{\circ} \mathrm{C}-34^{\circ} \mathrm{C}\right)$. Under cardioplegic arrest the branches of the right and circumflex coronary arteries were anastomosed to the free right ITA or radial artery. The left ITA was then used for revascularization of the anterior descending system. Sequential anastomoses were performed with both grafts as necessary. The T anastomosis between left and right ITA or radial artery was created during the period of cardiac arrest.

Coronary angiography protocol. All patients were offered the option of coronary angiography 1 week after the operation to document the adequacy of the surgical result. In 142 patients $(56.6 \%)$ informed consent was obtained and follow-up angiography performed.

During the course of our clinical experience, a clinical investigation was designed to assess flow characteristics of the $\mathrm{T}$ graft. The protocol was approved by the local ethics committee. Thirty-five patients with T grafts of the right ITA 
Table III. Operative data of patients with $T$ grafts

\begin{tabular}{lccl}
\hline & $\begin{array}{c}\text { LITA-RITA } \\
(n=73)\end{array}$ & $\begin{array}{c}\text { LITA-RA } \\
(n=178)\end{array}$ & P value \\
\hline Time & & & \\
$\quad$ Operation (min) & $244 \pm 44$ & $195 \pm 41$ & $<.0001$ \\
Bypass (min) & $91 \pm 26$ & $89 \pm 27$ & $>.2$ \\
$\quad$ Ischemia (min) & $64 \pm 22$ & $65 \pm 16$ & $>.2$ \\
Postop IABP (n) & $1(1 \%)$ & $3(2 \%)$ & $>.2$ \\
Concomitant procedures & & & \\
AVR (n) & $1(1 \%)$ & $4(2 \%)$ & $>.2$ \\
MVR (n) & 0 & $2(1 \%)$ & $>.2$ \\
Bullectomy (n) & 0 & $1(1 \%)$ & $>.2$ \\
Carotid TEA (n) & 0 & $3(2 \%)$ & $>.2$ \\
\hline
\end{tabular}

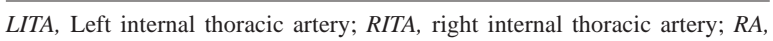
radial artery; $I A B P$, intra-aortic balloon pump; $A V R$, aortic valve replacement; $M V R$, mitral valve repair; $T E A$, thromboendarterectomy.

to the left ITA (group I) and 57 with T grafts of the left ITA and radial artery (group II) gave their informed consent for additional flow measurement in the left ITA 1 week after the operation. Six months later 18 group I patients and 10 group II patients were restudied by means of the same protocol. The statistical comparison of flow data over time was performed in these 28 patients. Their clinical characteristics, especially coronary risk factors with potential influence on their bypass flow measurements, are shown in Table II.

Catheterization technique. Administration of all medication was terminated 24 hours before cardiac catheterization. Selective angiography of the coronary arteries by the Judkins technique with $6 \mathrm{~F}$ diagnostic catheters was performed from a femoral approach with a $6 \mathrm{~F}$ sheath. Before the grafts were visualized, $0.2 \mathrm{mg}$ of glycerol trinitrate was applied into the left ITA.

Quantitative angiographic analysis. Quantitative analysis of the grafts was performed with an automated coronary analysis program with edge contour detection (CAAS II; Pie Medical, Maastricht, The Netherlands). The first well-opacified end-diastolic frame detected by simultaneous electrocardiographic recording was selected for analysis. The luminal diameter of the vessel at the level of blood velocity recordings was measured by an automated contour detection algorithm. Absolute dimensions were calculated by reference to the known size of the shaft of the empty diagnostic catheter, measured 2 to $3 \mathrm{~cm}$ from the tip. The cross-sectional area of the graft was then computed. For this purpose a circular cross section was assumed. The exact location of the lumen measurement 1 week after the operation was documented with a photographic printout. Six months later, the luminal diameter was measured at exactly the same location of the proximal left ITA.

Flow measurements. Phasic coronary flow velocity was recorded in the proximal left ITA with the use of a 0.014inch, 12-MHz Doppler guide wire (FloWire; Cardiometrics, Inc, Mountain View, Calif). The tip of the wire was placed exactly at the site where the luminal diameter had been mea-
Table IV. Patterns of arrangement of conduits of patients with $T$ grafts

\begin{tabular}{lcc}
\hline & $\begin{array}{c}\text { LITA-RITA } \\
(n=68)\end{array}$ & $\begin{array}{c}\text { LITA-RA } \\
(n=178)\end{array}$ \\
\hline Anastomoses/patient (n) & $4.0 \pm 0.9$ & $4.3 \pm 0.8$ \\
Distal anastomoses (n) & 299 & 771 \\
LITA (n) & $123(41 \%)$ & $287(37 \%)$ \\
RITA (n) & $176(59 \%)$ & - \\
RA (n) & - & $484(63 \%)$ \\
Vein graft on LAD (n) & 0 & $2(1 \%)$ \\
\hline
\end{tabular}

LITA, Left internal thoracic artery; RITA, right internal thoracic artery; $R A$, radial artery; $L A D$, left anterior descending coronary artery.

sured before. An optimal Doppler signal was obtained by moving the guide wire slightly into the vessel lumen and adjusting the velocity range. The final position of the guide wire was confirmed by injection of contrast medium. Frequency analysis of the Doppler signal was carried out in real time by fast Fourier transformation with the use of a velocimeter (FloMap, Cardiometrics, Inc). After Doppler signals were recorded under baseline conditions, adenosine (30 $\mu \mathrm{g}$ ) was injected into the left ITA graft. The Doppler signals were recorded for 1 minute after injection to obtain the maximal peak velocity. Systolic and diastolic peak velocities and the time average of the instantaneous spectral peak velocity (time-average peak velocity) were determined from phasic coronary blood flow recordings. The flow volumes in the left ITA graft were calculated as proposed by Doucette and associates, ${ }^{12}$ using the mean velocity and the cross-sectional area. The CFR was obtained from the ratio of maximal to baseline flow.

Statistics. Data were analyzed with the use of the StatView software package (Abacus Concepts, Inc, Berkeley, Calif). All data are expressed as mean values \pm standard deviation. Clinical data of the 2 groups were compared by means of the unpaired $t$ test and $\chi^{2}$ tests.

The Wilcoxon signed rank test was used for analysis of flow data in each group. The 2 groups were compared by means of the $\mathrm{F}$ test for equal variances.

\section{Results}

Clinical results. A $\mathrm{T}$ graft of the right ITA to the left ITA was used in $73(29 \%)$ patients whereas $178(71 \%)$ patients received a combination of left ITA and radial artery. Operative data of these patients are shown in Table III. Implantation of an intra-aortic balloon pump for low cardiac output syndrome was necessary in $1.4 \%$ in group I and $1.7 \%$ in group II.

A mean of 4.0 (group I) versus 4.3 (group II) anastomoses were performed per patient (Table IV). In 2 patients of group II who could not be weaned from bypass, transesophageal echocardiography showed hypokinesia of the anterior wall. An additional vein 
Table V. Clinical outcome of patients with $T$ grafts

\begin{tabular}{|c|c|c|c|}
\hline & $\begin{array}{c}\text { LITA-RITA } \\
(n=73)\end{array}$ & $\begin{array}{c}\text { LITA-RA } \\
(n=178)\end{array}$ & $\mathrm{P}$ value \\
\hline Bleeding/reoperation (n) & 0 & $1(1 \%)$ & $>.2$ \\
\hline Myocardial infarction (n) & $2(2 \%)$ & $4(2 \%)$ & $>.2$ \\
\hline Phrenic nerve injury (n) & $1(1 \%)$ & 0 & $>.2$ \\
\hline Cerebral infarction (n) & 0 & $4(2 \%)$ & .2 \\
\hline \multicolumn{4}{|l|}{ Sternum } \\
\hline Dehiscence (n) & 0 & $1(1 \%)$ & $>.2$ \\
\hline Infection (n) & 0 & $1(1 \%)$ & $>.2$ \\
\hline \multicolumn{4}{|l|}{ Forearm } \\
\hline Circulatory injury (n) & - & 0 & - \\
\hline Infection (n) & - & $2(1 \%)$ & - \\
\hline Paresthesia (n) & - & $14(8 \%)$ & - \\
\hline In-hospital mortality (n) & $2(2.7 \%)$ & $4(2.3 \%)$ & $>.2$ \\
\hline
\end{tabular}

LITA, Left internal thoracic artery; RITA, right internal thoracic artery; $R A$, radial artery.

graft was placed on the left anterior descending coronary artery. With restored anterior wall function both patients could be weaned from bypass and were finally discharged after an unremarkable postoperative course.

The in-hospital mortality was acceptable in both groups (group I, 2.7\%; group II, 2.3\%; Table V). Four of the 6 deaths were cardiac. In group I a man with concomitant colon cancer had a significant intraoperative myocardial infarction and could not be weaned from cardiopulmonary bypass. Two female patients in group II had an uneventful operation with stable postoperative hemodynamics. Several hours later cardiac fibrillation suddenly developed and they underwent cardiopulmonary resuscitation including circulatory support with extracorporeal circulation. Placement of an additional vein graft to the left anterior descending coronary artery was unsuccessful in restoring left ventricular function. The pathologic investigation documented patent grafts. One woman from group II, who underwent reoperation and concomitant mitral valve repair, had postoperative low cardiac output syndrome and died 3 days later of multiorgan failure.

Angiographic results. One week after the operation the patency rate of the left ITA was $98.1 \%$ in group I $(53 / 54)$ and $98.9 \%(87 / 88)$ in group II. The free right ITA was patent in $94.4 \%(51 / 54)$ and the radial artery in $97.7 \%(86 / 88)$ of patients.

In the group of patients who underwent angiography 6 months after the operation, 1 radial artery (of 10) was occluded in the presence of significant perfusion via the native coronary system.

Flow measurement results. Baseline flow 1 week after the operation was $76.13 \pm 34.08 \mathrm{~mL} / \mathrm{min}$ in group $\mathrm{I}(\mathrm{n}=35)$ and $72.65 \pm 34.78 \mathrm{~mL} / \mathrm{min}$ in group II $(\mathrm{n}=$
57) $(P=.9)$. After stimulation with adenosine, the flow increased in both groups (group I, $136.34 \pm 53.43$ $\mathrm{mL} / \mathrm{min}, P<.0001$; group II, $130.83 \pm 58.80 \mathrm{~mL} / \mathrm{min}$, $P<.0001)$. There was no significant difference in the maximum flow between the 2 groups $(P=.5)$. The calculated CFR was $1.87 \pm 0.37$ (group I) versus $1.86 \pm$ 0.33 (group II) $(P=.5)$.

In the patients who were investigated 6 months later (group I, $\mathrm{n}=18$; group II, $\mathrm{n}=10$ ), the baseline flow was slightly reduced in both groups (Fig 1; group I, $P$ $=.03$; group II, $P=.9$ ) without significant difference between the 2 groups $(P=.3)$. Medical stimulation with adenosine resulted in a flow elevation (Fig 1; group I, $P=.0002$; group II, $P=.005$ ). The maximum flow showed no significant difference between the 2 groups $(P=.7)$. Over time, the maximum flow increased mildly compared with that measured 1 week after the operation (Fig 1; group I, $P=.9$; group II, $P=$ .1). Although there was no significant difference between the 2 groups $(P=.9)$, the CFR elevation over time was significant in each group (Fig 1; group I, $P=$ .0002 ; group II, $P=.009$ ).

\section{Discussion}

Short-term and long-term data have long confirmed the importance of the use of at least 1 arterial graft in CABG. Several investigations have found improved early postoperative recovery. ${ }^{13}$ Likewise the long-term prognosis resulting from freedom from angina and decreased mortality rates has been improved by placing 1 ITA to the left anterior descending coronary artery. ${ }^{1,3}$ In view of the excellent long-term function of the ITA, the current trend is to expand the role of arterial grafts in CABG further. Although some reports deny the benefit of using more than 1 ITA graft, ${ }^{14}$ a number of investigations have documented improved long-term results after using 2 ITAs when placed to the anterior descending and circumflex arteries. ${ }^{2,4,5}$ These promising results have stimulated efforts to achieve complete arterial revascularization to eliminate or minimize the effect of graft degeneration in coronary operations. Different combinations using a $\mathrm{T}$ graft of the right ITA to the left ITA alone or in combination with other arteries are used.

The radial artery as a conduit for CABG was first proposed and used by Carpentier and associates. ${ }^{15}$ Recently, the radial artery was reintroduced as a bypass graft by Acar, ${ }^{7}$ Calafiore, ${ }^{8}$ and their associates. Although there is a higher propensity to spasm of the radial artery than of the ITA, ${ }^{16}$ excellent results with respect to early patency and morbidity in CABG have been reported for the radial artery., ${ }^{77}$ The gastroepiploic artery has also been used as an alternative arteri- 


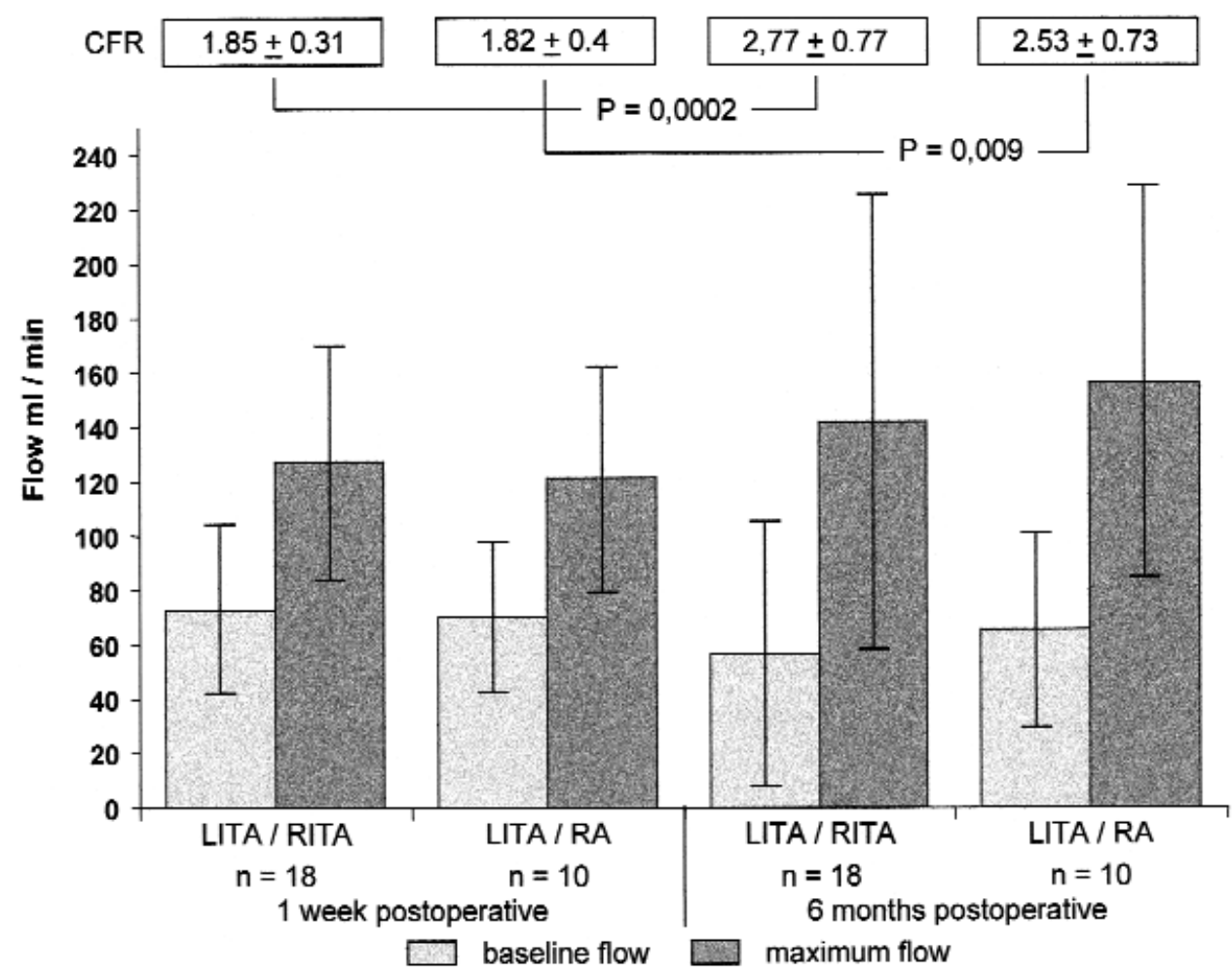

Fig 1. Results of flow measurements in the left ITA main stem. CFR, Coronary flow reserve; LITA, left internal thoracic artery; RITA, right internal thoracic artery; $R A$, radial artery.

al conduit in CABG. ${ }^{18}$ The risk of vasospasm is increased in relation to the ITA. Results with the gastroepiploic artery are comparable with those with the radial artery in the early postoperative years. ${ }^{7,8,17,19,20}$ The radial artery appears to have advantages over the gastroepiploic artery because it can be harvested while the left ITA is being dissected. Surgical dissection appears easier, and a laparotomy with its associated morbidity can be avoided. Commonly, 3 or more of these arterial grafts are used to achieve total revascularization. Medium-term results of complete arterial revascularization appear promising. ${ }^{21}$

$\mathrm{T}$ grafts, as suggested by Tector and associates, ${ }^{6}$ achieve the goal of complete arterial revascularization with only 2 arterial grafts. Originally the left ITA is anastomosed to coronary branches of the anterior descending coronary artery. The free right ITA is connected to posterolateral and inferior branches of the circumflex and right coronary artery in sequential fashion. Because of its limited length, the proximal anastomosis of the right ITA is created with the left ITA. Other authors have propagated the T-graft procedure with the gastroepiploic or radial artery being used as the second graft, and good perioperative results have been reported. ${ }^{7,9,17}$
With the T-graft technique, complete arterial revascularization in triple vessel disease is possible with only 2 grafts. The economic application of grafts may play a role in young patients with further progress of the coronary disease and resulting need for late reoperation. Trauma and dissection time is shortened compared with that of multiarterial harvesting. Avoidance of a proximal anastomosis to the aorta is particularly beneficial in patients with calcification of the ascending aorta. A potential disadvantage of this approach may result from the fact that coronary bypass flow is totally dependent on the flow in the proximal ITA. Reduction of flow in the left ITA, caused by vasospasm or trauma, may result in a hypoperfusion syndrome with global ischemia and its catastrophic consequences. ${ }^{22}$ In addition, the procedure is technically more difficult than CABG using the left ITA and vein grafts. Particularly patients with small graft diameters, like the 2 women in this series who had ITA hypoperfusion syndrome several hours after the operation, may be at an increased risk of surgical pitfalls. From the fatalities in our limited experience it is difficult to judge whether the higher propensity of radial arteries in 3 of 4 patients may have contributed.

To reduce the risk of sternal wound infection after 
using both ITAs, ${ }^{23}$ we have employed the skeletonized ITA preparation technique described by Cunningham and associates. ${ }^{24}$ We have furthermore found that free flow of the skeletonized ITA was better than that of ITAs harvested in a pedicle after intraluminal application of papaverine. ${ }^{11}$ Although initial $\mathrm{T}$ grafts were sometimes technically cumbersome to construct, we were impressed by the ease of the operation with skeletonized ITAs. We have thus used skeletonized arterial grafts routinely for CABG. The risk of vasospasm and hypoperfusion syndrome of the ITA appears low with our approach, which is reflected in the low incidence of intra-aortic balloon pump implantation and mortality rates in our population.

Since principal concern with respect to flow limitations still remained valid, we decided to explore this question under strict conditions of a clinical study. Patients who underwent the T-graft procedure and follow-up angiography 1 week after the operation had excellent patency rates.

Intravascular flow measurements with a Doppler guide wire in the left ITA main stem at rest and after peripheral vasodilation with adenosine were performed after documentation of an excellent surgical result. In the absence of significant changes in heart rate and ventricular preload, CFR measurements (ratio of peak hyperemic to resting coronary blood flow velocity) are highly reproducible in human beings..$^{25}$ The intravascular Doppler measurement is a valuable clinical tool in the longitudinal assessment of the coronary circulation. In 1995, 2 investigations about flow measurement in single ITA grafts were published. ${ }^{26,27}$ The maximal blood flow in these studies was stimulated with papaverine/isosorbide nitrate ${ }^{27}$ and dipyridamole. ${ }^{26}$ Both groups showed reduced CFR in the first days after operation, although CFR increased over time.

In the present study both cohorts of patients showed a slightly elevated baseline flow 1 week after the operation, which might be explained by early postoperative reduction in hemoglobin with the elevation of cardiac index. In combination with an increased maximum flow after 6 months, a significant elevation of the CFR after 6 months was observed. In both groups we found no significant differences between the patients operated on with either a free right ITA-left ITA or a left ITA-radial artery $\mathrm{T}$ graft because of baseline flow, maximum flow, and CFR 1 week and 6 months after the operation. It is remarkable that baseline flow in the left ITA main stem was higher after $\mathrm{T}$ grafting than flow rates in single ITA grafts, which have been reported to range between 30 and $40 \mathrm{~mL} / \mathrm{min} .{ }^{27}$ This higher flow in the left ITA reflects the greater supported myocardial area. The CFR and its dynamic over time is comparable with that after single ITA grafting. ${ }^{27}$ Therefore one can hypothesize that the T-graft configuration guarantees adequate blood supply of the anastomosed myocardium with nearly normal CFR.

These results have prompted us to offer complete arterial revascularization to all patients less than 70 years of age. Currently the percentage of complete arterial procedures in our clinic is in excess of $40 \%$. With the documentation of these hemodynamic results, which are identical for left ITA-right ITA versus left ITA-radial artery configuration, we conclude that this operation can be applied on a routine basis, allowing for complete arterial revascularization of a large proportion of patients.

\section{REFERENCES}

1. Cameron A, Kemp HG, Green GE. Bypass surgery with the internal mammary artery graft: 15 year follow-up. Circulation 1986: 74(Suppl):III30-6.

2. Fiore AC, Naunheim KS, Dean P, Kaiser GC, Pennington G, Willman VL, et al. Results of internal thoracic artery grafting over 15 years: single versus double grafts. Ann Thorac Surg 1990;49:202-9.

3. Loop FD, Lytle BW, Cosgrove DM, Stewart RW, Goormastic M, Williams GW, et al. Influence of the internal mammary artery graft on 10-year survival and other cardiac events. N Engl J Med 1986;314:1-6.

4. Dewar LRS, Jamieson E, Janusz MT, Adeli-Sardo M, Germann E, MacNab JS, et al. Unilateral versus bilateral internal mammary revascularization. Circulation 1995:92(Suppl):II8-13.

5. Pick AW, Orszulak TA, Anderson B, Schaff HV. Single versus bilateral internal mammary artery grafts: 10-year outcome analysis. Ann Thorac Surg 1997;64:599-605.

6. Tector AJ, Amundsen S, Schmahl TM, Kress DC, Peter M. Total revascularization with T grafts. Ann Thorac Surg 1994;57:33-9.

7. Acar C, Jebara VA, Porthoghese M, Beyssen B, Pagny JY, Grare $\mathrm{P}$, et al. Revival of the radial artery for coronary artery bypass grafting. Ann Thorac Surg 1992;54:652-60.

8. Calafiore AM, Giammarco GD, Luciani N, Maddestra N, Nardo $\mathrm{ED}$, Angelini R. Composite arterial conduits for a wider arterial myocardial revascularization. Ann Thorac Surg 1994;58:185-90.

9. Barner HB. Arterial grafting: techniques and conduits. Ann Thorac Surg 1998;66:S2-5.

10. Buxton B, Fuller J, Gaer J, Liu JJ, Mee J, Sinclair R, et al. The radial artery as a bypass graft. Curr Opin Cardiol 1996;11:591-8.

11. Wendler O, Tscholl D, Huang Q, Schäfers HJ. Free flow capacity of skeletonized versus pedicled internal thoracic artery grafts in CABG. Eur J Cardiothorac Surg 1999;15:247-50.

12. Doucette JW, Corl PD, Payne HM, Flynn AE, Goto M, Nassi M, et al. Validation of a Doppler guide wire for intravascular measurement of coronary artery flow velocity. Circulation 1992;85: 1899-911.

13. Edwards FH, Clark RE, Schwartz M. Impact of internal mammary artery conduits on operative mortality in coronary revascularization. Ann Thorac Surg 1994;57:27-32.

14. Sergeant PT, Blackstone EH, Meyns BP. Does arterial revascu- 
larization decrease the risk of infarction after coronary artery bypass grafting? Ann Thorac Surg 1998;66:1-11.

15. Carpentier A, Guermonprez JL, Deloche A, Frechette C, DuBost C. The aorta-to-coronary radial artery bypass graft. Ann Thorac Surg 1973;16:111-21.

16. Chardigny C, Jebara VA, Acar C, Descombes JJ, Verbeuren TJ, Carpentier A, et al. Vasoreactivity of the radial artery. Circulation 1993;88(pt 2):115-27.

17. Calafiore AM, Giammarco GD, Teodori G, Annunzio ED, Vitolla G, Fino C, et al. Radial artery and inferior epigastric artery in composite grafts: improved midterm angiographic results. Ann Thorac Surg 1995;60:517-40.

18. Mills NL. Arterial grafts for coronary artery bypass. Adv Card Surg 1997;9:195-216.

19. Suma H, Wanibuchi Y, Terada Y, Fukuda S, Takayama T, Furuta $\mathrm{S}$. The right gastroepiploic artery graft. J Thorac Cardiovasc Surg 1993;105:615-23.

20. Tatoulis J, Buxton BF, Fuller JA. Bilateral radial artery grafts in coronary reconstruction: techniques and early results in 261 patients. Ann Thorac Surg 1998;66:714-20.

21. Bergsma TM, Grandjean JG, Voors AV, Boonstra PW, den Heyer $\mathrm{P}$, Ebels T. Low recurrence of angina pectoris after coronary artery bypass surgery with bilateral internal thoracic and right gastroepiploic arteries. Circulation 1998;97:2402-5.

22. Jones EL, Lattouf OM, Weintraub WS. Catastrophic consequences of internal mammary artery hypoperfusion. J Thorac Cardiovasc Surg 1989;98:902-7.

23. Bical O, Braunberger E, Fischer M, Robinault J, Foiret JC, Fromes Y, et al. Bilateral skeletonized mammary artery grafting: experience with 560 consecutive patients. Eur J Cardiothorac Surg 1996;10:971-6.

24. Cunningham JM, Gharavi MA, Fardin R, Meek RA. Considerations in the skeletonization technique of internal thoracic artery dissection. Ann Thorac Surg 1992;54:947-51.

25. McGinn AL, White CW, Wilson RF. Interstudy variability of coronary flow reserve. Circulation 1990;81:1319-30.

26. Akasaka T, Yoshikawa J, Yoshida K, Maeda K, Hozumi T, Nasu M, et al. Flow capacity of internal mammary artery grafts: early restriction and later improvement assessed by Doppler guide wire. J Am Coll Cardiol 1995;25:640-7.

27. Gurne O, Chenu P, Polidori C, Louagie Y, Buche M, Haxhe JP, et al. Functional evaluation of internal mammary artery bypass grafts in the early and late postoperative periods. J Am Coll Cardiol 1995;25:1120-8.

\section{Discussion}

Dr Alfred J. Tector (Milwaukee, Wis). We have performed $\mathrm{T}$ grafts for total ITA revascularization in more than 1000 patients. The main concern of the T-graft technique is whether the attached left ITA adequately perfuses the entire ischemic myocardium. The authors report their results with the T-graft technique in 251 patients undergoing CABG. The right ITA and left ITA combination was used in 73 patients, and the radial artery from the nondominant arm combined with the left ITA was selected in 178 patients. An average of 4 or more arterial-coronary artery anastomoses were constructed in each patient.

The phasic coronary flow velocity in the proximal left ITA was measured with a Doppler guide wire in 92 patients at 1 week and in 28 patients 6 months after the operation. CFR was equal in both the right ITA-left ITA combination and the radial artery-left ITA group at 1 week and 6 months after the operation. At 6 months it reached near normal levels. This is the first time that CFR has been measured and reported postoperatively in a $\mathrm{T}$ graft.

These hemodynamic measurements demonstrate that flow in the attached left ITA is sufficient to supply the entire ischemic myocardium. This is convincing evidence for those of us who were interested in this technique.

Operative mortality was acceptable and graft patency studied 1 week and 6 months after the operation was excellent. Sternal dehiscence and wound infections occurred in 2 of the patients receiving the radial artery-left ITA combination; also 2 patients in this group had arm infections, and 14 had paresthesias in the forearm. One patient in the left ITA-right ITA group had phrenic nerve injury. Hypoperfusion was reported in 4 patients in the radial artery-left ITA group, and 2 of these persons died.

The authors describe performing the right ITA and radial artery-coronary anastomosis before doing the $\mathrm{T}$ graft. We believe the $\mathrm{T}$ anastomosis should be constructed first. Besides reducing cardiopulmonary bypass time, it allows the surgeon to assess flow in each limb of the graft, and if it is inadequate, the $\mathrm{T}$ anastomosis can be reconstructed, usually with very good flows in the limbs. Also, if there is an extra artery and it needs to be excised, it is excised from the smaller, less desirable distal end of the graft instead of the proximal end.

I have four questions for Dr Wendler.

Do you think it may be beneficial to measure critical flow in the left ITA distal to the T anastomosis?

Do you know how many radial arteries and how many ITAs were unacceptable at the time of grafting?

Were calcium channel blockers used?

Which combination of $\mathrm{T}$ graft, either radial artery-left ITA or right ITA-left ITA will be your first choice in the future?

Dr Wendler. Measurement of flow velocity distal to the T graft involves a technical problem to push the guide wire through the T graft in the peripheral vessel of the left ITA off the radial artery. Our cardiologists have been reluctant to perform this maneuver.

The second question deals with the radial arteries that were not accepted during the operation. By skeletonizing both ITAs, we had only 1 right ITA that was not acceptable for operation, and we had to operate on this patient with a vein graft. In all other patients, after skeletonization of the ITA, the graft was acceptable and showed an acceptable free flow during the operation.

Intraoperatively, 7 patients were found to have calcification in the radial artery, and we decided to use the right ITA for the T-graft configuration. Preoperatively, 10 patients had a pathologic Allen test and were not acceptable for radial artery harvesting.

The third question concerns calcium channel blockers. We do not use calcium channel blockers in these patients intravenously, but after harvesting of the radial artery, we sprayed 
the radial arteries externally with diltiazem after administering papaverine intraluminally.

The fourth question concerns which combination might be the best for the patients. As we have seen, the operation time is significantly reduced with the left ITA-radial artery configuration. This is a result of the fact that harvesting of the left radial artery is possible during the dissection of the left ITA graft. Our general approach is to use the left ITA and radial artery of the nondominant arm in the patient with an acceptable Allen test. If calcification of the radial artery, for example, is detected during the operation, we have the option of changing to the left ITA-right ITA configuration.

Dr Bruce W. Lytle (Cleveland, Ohio). Were the T anasto- moses constructed as true $\mathrm{T}$ anastomoses, that is to say, at right angles, or were they constructed in what some people would term a $\mathrm{Y}$ anastomosis, a more acute angle?

Dr Wendler. Thank you very much for this good question. As Dr Tector said, we performed the T-graft anastomosis after we had completed all distal anastomoses. We have not decided which configuration of this anastomosis yields the optimal surgical result. In many patients the result is a real T configuration, but in some patients the $\mathrm{Y}$ configuration seems to have the better result.

Dr Lytle. Does the structure of your data make it possible for you to interpret whether there is any difference in those constructions?

Dr Wendler. No, we have no data about that.

\section{Bound volumes available to subscribers}

Bound volumes of The Journal of Thoracic and Cardiovascular Surgery are available to subscribers (only) for the 1999 issues from the Publisher, at a cost of $\$ 134.00$ for domestic, \$165.85 for Canadian, and \$155.00 for international subscribers for Vol 117 (January-June) and Vol 118 (July-December). Shipping charges are included. Each bound volume contains a subject and author index and all advertising is removed. Copies are shipped within 60 days after publication of the last issue of the volume. The binding is durable buckram with the Journal name, volume number, and year stamped in gold on the spine. Payment must accompany all orders. Contact Mosby, Inc, Subscription Services, 11830 Westline Industrial Drive, St Louis, MO 63146-3318, USA; phone 800-453-4351 or 314-453-4351.

Subscriptions must be in force to qualify. Bound volumes are not available in place of a regular Journal subscription. 